Finally, no contemporary review of this subject should omit a reference to the very important article by Klein et $a l^{4}$ on "Large artery involvement in giant-cell (temporal) arteritis."

Ipswich

J W Paulley

'Hutchinson, J, Archives of Surgery (London), 1890 1, 323. Horton, B T, Magath, T B, and Brown,
ceedings of the Mayo Clinic, 1932, 7, 700 . ceedings of the Mayo Clintc, $1932,7,700$.
Ali Ibn Isa, Memorandum of a 10 th Century Oculist, translated by C A Woods, Chicago NW University, 1936. Cited by Hamilton, C R, Shelley, W M, and Tumulty, P A, Medicine, $1975, \mathbf{5 0}$, Klein, $\mathrm{R}$ G, et al, Annals of Internal Medicine, 1975,
83, 806.

** ${ }^{*}$ Dr Paulley is correct. Jonathan Hutchinson did describe one case, in an elderly porter at the London Hospital, in 1890, before Horton in 1932, just as others had described what Barber later termed polymyalgia rheumatica in 1957, but in each case the fuller description merited the attention it attracted. Dr Paulley is also correct in saying that the ophthalmic artery and its other branches become occluded more often than the central artery of the retina. Wilkinson and Russell $^{1}$ in their necropsy study of 12 patients who died with active giant-cell arteritis found a high incidence of disease of the superficial temporal, vertebral, ophthalmic, and posterior ciliary arteries with less frequent disease of the internal and external carotid and central retinal arteries.-ED, $B M \mathcal{H}$.

Wilkinson, I M S, and Russell, R W R, Archives of Neurology, 1972, 27, 378 .

SIR,-Although there is considerable overlap between polymyalgia rheumatica and giant cell arteritis it is doubtful whether arteritis is the underlying pathological process in the former (leading article, 23 April, p 1046). There is histological $^{1}$ and joint scintigraphic ${ }^{2}$ evidence that a proximal joint synovitis accounts for the symptoms of polymyalgia rheumatica.

This is not an academic point. Although the symptoms of polymyalgia rheumatica respond to low doses of prednisolone, these are inadequate to prevent vascular occlusion from arteritis and there therefore seems no reason why anti-inflammatory analgesics should no be tried first. The large doses of corticosteroid necessary to treat arteritis have serious, unwanted effects in the elderly and should be administered only when there is clinical or histological evidence of an arteritis.

On practical therapeutic grounds at least there therefore seems reason to retain the distinction between giant cell arteritis and polymyalgia rheumatica and not to combine them as polymyalgia arteritica.

D R SwINSON

Rheumatology Unit,

Wrightington Hospital

Wigan, Lancs

1 Bruk, M I, Annals of the Rheumatic Diseases, 1967, 26

' O'Duffy, J D, Wahner, H W, and Hunder, G G, Mayo Clinic Proceedings, 1976, 51, 519.

SIR,-May I be allowed to comment on your very good leading article about polymyalgia rheumatica (23 April, p 1046)?

In my experience it is often necessary to give between 45 and $60 \mathrm{mg}$ of prednisolone daily to control morning stiffness and girdle pains; it may well be advisable to begin treatment with a smaller dose if there is any evidence of cardiovascular disease.
Loss of vision may occur very suddenly without an increase of symptoms and without a spread of symptoms to affect the temples or scalp. I myself have missed a case of polymyalgia with arteritic lesions which produced blindness. The patient was a proven rheumatoid who complained of sudden increase of pain in both shoulders. As her erythrocyte sedimentation rate (ESR) was only marginally raised at $18 \mathrm{~mm}$ in $1 \mathrm{~h} \mathrm{I}$ dismissed the diagnosis of polymyalgia even though I thought of it. She returned to the clinic the following week blind in one eye, a temporal artery biopsy proving positive. Involvement of the facial artery occurs occasionally, such patients often being referred to an ear, nose, and throat surgeon.

Lastly, I have encountered polymyalgia apparently as a systemic manifestation of a neoplasm; one useful guide here is that whereas symptoms abate with steroids the ESR remains very high.

I think that polymyalgia is the only condition in which I prescribe the appropriate drug from a hospital clinic. Any such patient is at risk from arteritis causing visual loss; it is my practice to ask the patient to remain in the clinic until the result of the ESR is to hand.

Harrogate Royal Bath Hospital,

JOHN R GOLDING

Harrogate, N Yorks

\section{Malignant hypertension secondary to} idiopathic arteritis of the aorta

SIR,-The very interesting case reported by Dr R A Wall and others (23 October, p 977) is the third such case to be reported from the Ndola Central Hospital, Zambia. ${ }^{12}$

The similarities between the patient reported by Dr Wall and his colleagues and our second one are very close. Both were young (16 and 20 years respectively) and had malignant renovascular hypertension, but renal function was well preserved and hypertension was easily controlled, in our case by drugs and in their case by nephrectomy and a diuretic. Their description of the histological appearance of the resected renal upper pole artery is very similar to our description of the aortic changes in the necropsy specimen in our first case-degeneration of the media.

Department of Medicine,

MARK N LOWENTHAL

wanath Hospital,

South Africa

' Lowenthal, $M$ N, Doctor, S A, and Fine, J, Post-

Cillai, K M, Lancet, 1971, 1, 295.

Absorption of lithium from controlledrelease preparations

SIR,-We have reported ${ }^{1}$ the 24 -h plasma lithium profiles of 18 fasting subjects who were given single oral doses of one or more of either the standard $B P$ or two controlled release preparations of lithium carbonate. Although the controlled-release tablets showed slow release in vitro, this was not so in vivo. There was no difference in the rate of absorption and excretion between the $B P$ preparation used (Camcolit) and one controlled-release preparation (Priadel), while the other slowrelease preparation (Phasal) was ineffectively absorbed by some subjects. Seven of the 18 subjects were normal volunteers who had never previously received lithium medication; this was also so for five of the 11 patients, while the other six patients had not received lithium for at least three months.

Although the shapes of the plasma lithium curves would of course be different if single doses of the various preparations were given to subjects stabilised on lithium, there is no reason to suppose that the rates of absorption and excretion would differ greatly under these conditions provided the single dose was administered not less than 10-12 h after previous lithium ingestion. However, in order to study these findings further under practical conditions we have investigated the effect of substituting the same daily dosage $(400 \mathrm{mg})$ of lithium carbonate $B P$ for one of the controlledrelease preparations (Priadel) in a group of 11 patients stabilised on the latter. Plasma lithium was determined on three occasions at weekly intervals before the substitution and again one, two, and three weeks after the switch, blood being taken in each case not less than $10 \mathrm{~h}$ after the last lithium dose. No significant differences between the pre- and postsubstitution mean plasma lithium levels were observed. The range of daily oral dosage was 400-2000 mg.

While the protocol of this investigation does not preclude the possibility of different diurnal plasma lithium patterns, the similar baseline levels obtained with the two preparations confirms our previous conclusion that there is no basis for administering Priadel in preference to lithium carbonate $B P$.

R P HULLIN

Regional Metabolic Research Unit,

High Royds Hospital,

Ilkley, W Yorks

' Tyrer, S, et al, Psychological Medicine, 1976, 6, 51.

\section{Predicting child abuse}

SIR,-The papers from the Park Hospital, Oxford, in the past two years have opened up the possibilities for helping families long before a disaster occurs.

Dr Margaret A Lynch and Mrs Jacqueline Roberts (5 March, p 624) are right in saying that "there seems to be a need for co-ordinating all disciplines, both in the hospital and in the community." This could be done by holding some antenatal examinations in the home. With the reorganisation of the Health Service community midwives are now based in hospitals in close contact with consultant units. When a midwife finds that a family scores highly on a number of factors she can discuss the significance of this with the family and alert the health visitor and family doctor.

Advantages of this suggestion are: (1) Prediction of child abuse in an informal way.

(2) Reduction of the size of antenatal clinics. After booking, antenatal checks in the early and middle months of pregnancy are medically dull and can be looked on as a waste of skilled obstetricians' time. Removal of about half these examinations would free the doctors for complicated situations where they are really needed. (3) Increase in the role, interest, and responsibility of community midwives. (4) Saving of money by transferring medical time from cost-intensive hospitals to the primary care team-generally found to be cheaper.

It is hoped that obstetricians, midwives, and paediatricians will collaborate, perhaps at 